\title{
Erratum to: Nebulized Magnesium Sulfate in Acute Bronchiolitis: A Randomized Controlled Trial
}

\author{
Mohammad Reza Modaresi ${ }^{1}$. Jamal Faghihinia ${ }^{2} \cdot$ Roya Kelishadi $^{2} \cdot$ Mohsen Reisi $^{1}$. \\ Shahrokh Mirlohi ${ }^{1} \cdot$ Farhad Pajhang $^{3} \cdot$ Majid Sadeghian $^{4}$
}

Published online: 16 March 2016

(C) Dr. K C Chaudhuri Foundation 2016

Erratum to: Indian J Pediatr 82(9):794-798

DOI 10.1007/s12098-015-1729-z

In the original article, the authors failed to cite the study by Kose $\mathrm{M}$ et al. entitled "The efficacy of nebulized salbutamol, magnesium sulfate and salbutamol/magnesium sulfate combination in moderate bronchiolitis. Eur J Pediatr 2014;173: $1157-1160 "$ as the first in literature to assess the efficacy of magnesium sulfate in the treatment of acute bronchiolitis. The authors apologize for this unintentional omission.

The online version of the original article can be found at http://dx.doi.org/ 10.1007/s12098-015-1729-z.

Mohsen Reisi

mohsenreisi72@yahoo.com

1 Department of Pediatric Pulmonology, Child Growth and Development Research Center, Research Institute for Primordial Prevention of Non-Communicable Disease, Isfahan University of Medical Sciences, Isfahan, Iran

2 Department of Pediatrics, Child Growth and Development Research Center, Research Institute for Primordial Prevention of Non-Communicable Disease, Isfahan University of Medical Sciences, Isfahan, Iran

3 Department of Pediatrics, Shariati Hospital of Isfahan, Isfahan, Iran

4 Department of Pediatrics, Tehran University of Medical Sciences, Tehran, Iran 